\title{
ACCIONES DE SANEAMIENTO CONTABLE EN LAS ENTIDADES GUBERNAMENTALES
}

\author{
SANITATION IN EQUITY SHARES OF GOVERNMENT INSTITUTIONS
}

\author{
${ }^{1}$ Pedro Ricardo Quiroz Quezada
}

\begin{abstract}
Resumen
Hoy en día en las entidades gubernamentales existe una problemática que se encuentra identificado en la calidad de la información de la Cuenta General de la República. Esta investigación se ha orientado al siguiente objetivo: Establecer como el saneamiento contable de los estados financieros podrá facilitar la calidad de la información de la Cuenta General de la República. Para lograr este objetivo la investigación se hizo mediante una encuesta y entrevista a 30 administrativos de una institución pública.El análisis de los resultados llega a las siguiente conclusión final: El saneamiento contable de los estados financieros facilita la calidad de la información de la Cuenta General de la Republica, mediante los ajustes y regularizaciones en el marco de las NIC-SP, Manual de estadísticas publicas, Clasificadores presupuestarios y Normas de control gubernamental.
\end{abstract}

Palabras clave: Saneamiento contable, estados financieros, calidad de la información, Cuenta General de la República, Sector Público.

\begin{abstract}
Today in government agencies there is a problem that is identified in the quality of information of the General Account of the Republic. This research has focused on the following objective: Set the accounting consolidation of financial statements may provide quality information to the General Account of the Republic. To achieve this goal the research was done through a survey and interviews with 30 staff of a public institution.

The analysis of the results reached the following conclusion: The accounting consolidation of financial statements provide the quality of information of the General Account of the Republic, through the settings and adjustments under IAS-SP, Handbook of Statistics public, budgetary rules Binders and government control.
\end{abstract}

Key words: Sanitation accounting, financial statements, quality of information, General Account of the Republic, Public Sector.

\section{Introducción}

La teoría contable dice que la Contabilidad es la herramienta de información por excelencia que facilita el proceso administrativo, como de la toma de decisiones internas y externas por parte de los diferentes usuarios; sin embargo cuando esta información no es exacta pierde su utilidad y confiabilidad y por el contrario puede inducir a decisiones equivocadas y antes de favorecer a las entidades puede perjudicarlas. Esto es lo que está pasando con la Cuenta General de la República, que al formularse con cierta información financiera que contiene errores en los registros contables no viene cumpliendo los objetivos para la cual fue concebida, no se formula de acuerdo a las normas actualmente vigentes. La falta de cumplimiento de los objetivos anteriores, hace que la Cuenta General de la República no cumpla otro elemental objetivo de facilitar la fiscalización de la actividad pública a través de información fidedigna que se espera tener al cierre de un ejercicio presupuestario. Según la Ley que aprueba la Cuenta General de la República, la aprobación de la misma, sólo deviene en un acto meramente formal de ordenamiento administrativo y jurídico que no implica la aprobación de la gestión ni los actos administrativos que aquella sustenta, los que serán objeto de acciones de control por parte de los órganos del Sistema Nacional de Control (SNC) y de fiscalización por el Poder Legislativo. Esta aseveración le quita trascendencia a este importante instrumento de información de la gestión del sector público lo cual ya es un problema. Una información que contiene errores, con observaciones que no han sido corregidas por la entidad no permitirá que esta puedan cumplir con su misión de organizar y conducir la gestión pública de acuerdo a sus competencias exclusivas, compartidas y delegadas, en el marco de las políticas nacionales y sectoriales, para contribuir al desarrollo integral y sostenible en la jurisdicción geográfica en que se encuentre. Como consecuencia de la auditoria efectuada por la Contraloría General de la República a la Cuenta General de la República, y haber observado diferentes calificaciones en los dictámenes de auditoria a los estados financieros de las entidades del sector público, el Congreso de la República solicitó al órgano rector de contabilidad evalúe la normativa que regule los aspectos relacionados con el saneamiento físico, legal y contable de los rubros que vienen afectando a los mencionados estados financieros. La Contraloría General 
de la República sustentó que la información contable de las entidades presentan las siguientes deficiencias: No se lleva a cabo la observación de inventarios físicos por los auditores; Falta de análisis de cuentas; Falta de rendición de cuentas, incluido el PNUD; Falta de registro de activos y pasivos; Terrenos registrados a Un Nuevo Sol; Reparaciones civiles con el Estado, no contabilizadas en su totalidad; Activos sin depreciar por falta de liquidaciones; Ministerios donde no se expresa opinión, por tener unidades ejecutoras en proceso de auditoria; Clasificaciones contables erradas; Saldos no conciliados entre entidades y empresas públicas.

El objetivo general de la investigación es establecer la manera como el saneamiento contable de los estados financieros podrá facilitar la calidad de la información de la Cuenta General de la Republica. Este objetivo general se desglosa en los siguientes objetivos específicos: a) Determinar como las Normas Internacionales de Contabilidad del Sector Publico (NIC SP) podrá facilitar la presentación adecuada de los resultados de la gestión publica de la Cuenta General de la Republica, b) Describir como las Normas de control gubernamental podrán facilitar el control y la fiscalización de la gestión publica en la Cuenta General de la Republica.

\section{Materiales y métodos}

\section{Tipo de Investigación.}

La presente investigación ha seguido las pautas de tipo descriptivo, ya que permite indagar y especificar aspectos y situaciones importantes que inciden en la necesidad de desarrollar la presente investigación.

\section{Diseño de la investigación}

El nivel de la investigación es correlacional, permite analizar el objetivo en la relación de cómo el saneamiento contable de los estados financieros podrá facilitar la calidad de la información de la Cuenta General de la Republica.

\section{Población y muestra.}

Se realizo una encuesta y se entrevisto a 30 personas del área administrativa/contable de una institución publica.

\section{Limitaciones del Estudio}

Esta investigación presenta las siguientes limitaciones:

a) La primera limitación es el lugar, únicamente se realizo en el departamento de Lima y dentro de Lima Metropolitana.

b) La segunda limitación es la obtención de la información por políticas del sector público.

c) Se ha tenido limitaciones en cuanto a recursos financieros, puesto que la investigación ha corrido a cargo del autor de esta investigación, en vista que el Ministerio de Economía y la Universidad Nacional Agraria la Molina no apoyan este tipo de investigaciones.

\section{Resultados y discusión ANTECEDENTES DE LA INVESTIGACIÓN}

Los antecedentes son los siguientes:

Hernández, D. (2007) Tesis: Desconcentración de la Cuenta General para facilitar el desarrollo, integral y sostenible de las regiones.

El autor señala que, de acuerdo con la normatividad sobre la Cuenta General de la República solo se ha previsto la formulación centralizada de este instrumento de información administrativa de la gestión del sector público que contiene información y análisis de la actuación de las entidades del Estado en el cumplimiento de sus objetivos durante un ejercicio presupuestario. Esta forma de manejo de la Cuenta General, no permite formular la información en forma oportuna, por tanto no es factible el cumplimiento de los objetivos de informar los resultados de la gestión de la administración del Estado en los diferentes procesos involucrados: económico, presupuestal, contable, financiero y metas; tampoco permite presentar el análisis cualitativo de la actuación de las entidades, especialmente de los gobiernos regionales; la falta de oportunidad, no permite proveer información para la toma de decisiones que facilite el proceso de planeamiento de programas y proyectos para el desarrollo económico y social del país, especialmente de las regiones geográficas y finalmente no es posible cumplir con facilitar la fiscalización del gobierno local, regional y local.

El Plan Contable Gubernamental 2009 (PCG 2009) que se presenta, recoge todo lo enunciado $\mathrm{y}$, además, los aportes y sugerencias de los profesionales contables independientes y representantes de las entidades directamente relacionadas con el quehacer contable, que fueran definidas como necesarias para su elaboración. El Plan Contable Gubernamental 2009, como herramienta de la contabilidad gubernamental y el Plan Contable General Empresarial permitirán mejorar de manera sustancial el registro y procesamiento de la información contable. Así como la formulación de los reportes exigidos en las rendiciones de cuentas para la elaboración de la Cuenta General de la República, las Cuentas Fiscales, las Cuentas Nacionales, el planeamiento, la fiscalización y el control, como también de los reportes de gestión que contarán con mayores niveles de desagregación y harán posible ampliar los niveles y la calidad del análisis contable como fundamento de la toma de decisiones, en las Unidades Ejecutoras y Pliegos del ámbito del Sistema Integrado de Administración Financiera (SIAF), el cual comprende al Gobierno Nacional, los Gobiernos Regionales y los Gobiernos Locales. En cuanto a las empresas del estado reportan información contable físicamente y a través del aplicativo informático via web diseñados para que informen a la Dirección General de Contabilidad Pública del Ministerio de Economía y Finanzas. 
La estructura del PCG 2009 comprende tres elementos de Balance: 1.- Activo, 2.- Pasivo y 3.- Patrimonio; tres elementos de Gestión: 4.- Ingresos, 5.- Gastos y 6.Resultado; un elemento de Presupuesto: 8.- Presupuesto y un elemento de Cuentas de Orden: 9.- Cuentas de Orden; a partir de los cuales se elaboraron los catálogos, la dinámica, los criterios de reconocimiento y medición y las referencias resumidas de las normas internacionales contables relacionadas para cada caso, con miras a facilitar su conocimiento, interpretación y aplicación en las transacciones contables de las entidades públicas que comprende el SIAF, para garantizar la transparencia, calidad y oportunidad de la información como medio de mejora de las decisiones de Estado.

El Plan Contable General Empresarial- PCGE, fue aprobado por el Consejo Normativo de Contabilidad, órgano del Sistema Nacional de Contabilidad que tiene como parte de sus atribuciones, el estudio, análisis y opinión sobre las propuestas de normas relativas a la contabilidad de los sectores público y privado, así como la emisión de las normas de contabilidad para las entidades del sector privado. Dentro de esas atribuciones, es que emite este Plan Contable General Empresarial (PCGE), que contempla los aspectos normativos establecidos por las Normas Internacionales de Información Financiera (NIIF).

\section{ANTECEDENTES NORMATIVOS:}

a. Constitución Política del Estado-1979: Establece en su Artículo 145 el Sistema Nacional de Contabilidad, el mismo que está compuesto por los recursos humanos, materiales, las entidades, procesos, técnicas y procedimientos.

b. Resolución Directoral No. 071-80-EFC/76.01 aprueba el Plan Contable Gubernamental del Sistema de ContabilidadGubernamental Integrada. Norma enmarcada dentro de las técnicas modernas de contabilización y que permitió efectuar el registro contable de las transacciones de las entidades del Gobierno adecuadamente y elaborar los estados financieros y presupuestarios, de manera que era compatible con las necesidades de desarrollo económico del país.

c. Ley No. 24680 de 03.06.1987: Ley del Sistema Nacional de Contabilidad. Esta norma determina las funciones, objetivos y estructura de los organismos y órganos componentes del Sistema Nacional de Contabilidad instituido por el Artículo 145 de la Constitución Política del Estado del año 1979; los mecanismos de coordinación de la contabilidad, así como las relaciones con otros organismos del sector público y privado.

d. Directiva No. 40-92-EF/93.11.1 del 05.10.1992: Simplificación de los procedimientos contables, modificación del Plan Contable Gubernamental y presentación de estados financieros. Esta norma tiene por finalidad señalar la documentación contable básica y los procedimientos de registro simplificados para la obtención de la información contable necesaria en las administraciones públicas (excepto empresas públicas). Esta norma establece los documentos fuente para el Área de Bienes: Orden de Compra - Guía de Internamiento, Póliza de Entrada de Bienes, Póliza de Salida de Bienes. Área de Fondos: Todo documento que constituye fuente de información para el registro contable, en el manejo de los fondos públicos se utiliza para sustentar los asientos contables en el libro obligatorio Caja y en los libros auxiliares de Bancos. Área de Tributación: Constituye documentos fuente aquellos que se utilizan para el registro contable en los libros Diario y Caja de las entidades captadoras de recursos financieros. Área de Endeudamiento: Constituye documentos fuente aquellos que sirven de base para el registro en el libro Diario y libro Caja de la Dirección General de Crédito Público. Área de Presupuesto: Aquellos documentos fuente que sustentan los recursos captados en el área de fondos y tributación y los documentos que sustentan la ejecución de gastos e inversiones en las reparticiones públicas (Recibo de Ingresos, Comprobantes de Pago, Planilla Única de Haberes, Valorizaciones de Obras, facturas, etc.)

Las causas que no han permitido el cumplimiento de los objetivos de la Cuenta general de la República son las siguientes:

- La deficiente aplicación de las Normas Internacionales de Contabilidad para el sector público

- Falta de utilización del Manual de Estadísticas de las Finanzas Públicas

- Falta de relación adecuada de los clasificadores presupuestarios y los rubros de los estados financieros

- Incumplimiento de las Normas de control gubernamental

De continuar esta situación no se logrará calidad en la información de la Cuenta General de la República, por cuanto no se podrá contar con los:

- Resultados de la gestión pública

- Análisis cuantitativo de las entidades públicas

- Información para el planeamiento institucional

- Control y fiscalización de la gestión pública

\section{TEORÍA DE LA CONTABILIDAD GUBERNAMENTAL}

Alvarado, J. (2013), señala que la Ley No. 28708Ley General del Sistema Nacional de Contabilidad, promulgada a los veinte días del mes de marzo de dos mil seis, son principios regulatorios de la Contabilidad Gubernamental o Contabilidad Pública los siguientes: i) Uniformidad: Establecer normas y procedimientos contables para el tratamiento homogéneo del registro, procesamiento y presentación de la información contable; ii) Integridad: Registro sistemático de la totalidad de los hechos financieros y económicos; iii) Oportunidad: Registro, procesamiento y presentación de 
la información contable en el momento y circunstancias debidas; iv) Transparencia: Libre acceso a la información, participación y control ciudadano sobre la contabilidad del Estado; v) Legalidad: Primacía de la legislación respecto a las normas contables.

De acuerdo con la ley, el Sistema Nacional de Contabilidad es el conjunto de políticas, principios, normas y procedimientos contables aplicados en los sectores público y privado. El Sistema Nacional de Contabilidad tiene los objetivos siguientes: a) Armonizar y homogeneizar la contabilidad en los sectores público y privado mediante la aprobación de la normatividad contable; b) Elaborar la Cuenta General de la República a partir de las rendiciones de cuentas de las entidades del sector público; c) Elaborar y proporcionar a las entidades responsables, la información necesaria para la formulación de las cuentas nacionales, cuentas fiscales y al planeamiento; y, d) Proporcionar información contable oportuna para la toma de decisiones en las entidades del sector público y del sector privado.

La investigación contable es el conjunto de actividades de estudio y análisis de los hechos económicos, financieros, su incidencia en la situación patrimonial, la gestión, los presupuestos y los costos en las entidades de los sectores público y privado, conducentes a la aprobación de normas y al mejoramiento de los sistemas y procedimientos contables.

La Dirección Nacional de Contabilidad Pública y el Consejo Normativo de Contabilidad, en el ámbito de sus competencias, dictan y aprueban normas y procedimientos contables aplicables en los sectores público y privado, respectivamente.

De acuerdo con la Ley, el registro contable es el acto que consiste en anotar los datos de una transacción en las cuentas correspondientes del plan contable que corresponda, utilizando medios manuales, mecánicos, magnéticos, electrónicos o cualquier otro medio autorizado y de acuerdo a lo establecido en la documentación que sustenta la transacción.

El registro contable que se efectúa utilizando el Sistema Integrado de Administración Financiera del Sector Público (SIAF-SP), tiene como sustento la Tabla de Operaciones cuya elaboración y actualización permanente es responsabilidad de la Dirección General de

Contabilidad Pública.

Las transacciones de las entidades registradas en los sistemas contables correspondientes, son clasificadas y ordenadas para la elaboración de los estados financieros, las notas a los estados financieros, de los estados presupuestarios e información complementaria de acuerdo a las normas contables vigentes.

\section{TEORÍA DE LA AUDITORIA DE LA CUENTA GENERAL DE LA REPÚBLICA}

Aldave, J. \& Meniz A. (2013), señalan que la Cuenta General de la República, es un instrumento de gestión pública que contiene información y análisis de los resultados presupuestarios, financieros, económicos, patrimoniales y de cumplimiento de metas e indicadores de gestión financiera durante un ejercicio fiscal. Para la elaboración de la Cuenta General de la República, todas las entidades del sector público se encuentran sujetas a la rendición de cuentas.

La auditoría de la Cuenta General de la República esta a cargo de la Contraloría General de la República. Al respecto, el Artículo $81^{\circ}$ de la Constitución Política del Estado, establece que la Cuenta General de la República, acompañada del informe de auditoría de la Contraloría General, es remitida por el Presidente de la República al Congreso en un plazo que vence el quince de noviembre del año siguiente al de ejecución del presupuesto. La Cuenta General es examinada y dictaminada por una Comisión Revisora dentro de los noventa días siguientes a su presentación. El Congreso se pronuncia en un plazo de treinta días. Si no hay pronunciamiento del Congreso en el plazo señalado, se eleva el dictamen de la Comisión Revisora al Poder Ejecutivo para que éste promulgue un decreto legislativo que contiene la Cuenta General.

La auditoría de la Cuenta General de la República, tiene como objetivos verificar si el contenido y estructura de la Cuenta General se adecua a lo establecido en la Ley General del Sistema Nacional de Contabilidad; verificar la confiabilidad y transparencia en la información y la aplicación correcta de los procedimientos establecidos en su elaboración; verificar la correcta integración y consolidación en los estados financieros. En el aspecto técnico la auditoria de la Cuenta General de la República se sustenta en auditorías financieras y presupuestales realizados por Sociedades de Auditoría, Órganos de Control Institucional, Contraloría General de la República. Dichos exámenes se realizan de conformidad con las Normas Internacionales de Auditoría, Normas de Auditoría Gubernamental -NAGU y Manual de Auditoría Gubernamental - MAGU, y demás normas aplicables.

Como resultado de la auditoría a la Cuenta General de la República, se emiten las conclusiones respectivas.

El efecto del total de calificaciones determinadas en los informes de la muestra, emitidos por los órganos del Sistema Nacional de Control (CGR, OCI y SOA), así como los reparos determinados en el marco de la auditoria a la Cuenta General de la República; con relación a los activos totales del Balance General y los ingresos del Estado de Gestión después de eliminaciones.

De acuerdo a lo que se señale en el Informe de Entidades Omisas a la Cuenta General de la República, la Contraloría General de la República realiza el análisis de las mismas, dando la recomendación pertinente a fin de disminuir la cantidad de las mismas a la presentación dela información para la Cuenta General.

A partir de la emisión de la Ley No. 29608, la Dirección General de Contabilidad Pública viene adoptando medidas para que las entidades del sector público efectúen el saneamiento de sus respectivas cuentas contables, 
emitiendo manuales de procedimientos, estableciendo la dinámica de las cuentas de saneamiento contable y ha elaborado un módulo de Saneamiento Contable a fin que las entidades registren las cuentas contables identificadas a sanear, reclasificar o corregir vía web.

Se sabe que 13 de julio de 2012 un total de 573 entidades del sector público se han acogido al proceso de saneamiento contable, lo que demuestra que el citado saneamiento se viene efectuando de manera progresiva, más aún con la implementación del Módulo de Saneamiento Contable vía Web, se espera se uniformice y acelere este proceso teniendo en cuenta la ampliación del plazo para la culminación de dicho proceso hasta el 31 de diciembre de 2012.

\section{TEORÍAS DEL SANEAMIENTO CONTABLE EN LOS ESTADOS FINANCIEROS}

Alvarado, J. (2013), señala que el saneamiento contable en los estados financieros gubernamentales es el acto de ajustar y/o regularizar los registros contables realizados por las entidades públicas en base a las Normas Internacionales de Contabilidad para el Sector Público.

El saneamiento contable en el sector público, debe llevarse a cabo en base a las siguientes normas legales: Ley N 29608 - Ley que aprueba la Cuenta General de la República del Ejercicio Fiscal 2009; Ley No 28708 - Ley General del Sistema Nacional de Contabilidad y modificatoria; Ley $\mathrm{N}^{\circ} 28112$ - Ley Marco de la Administración Financiera del Sector Público; Resolución Directoral No 001-2009-EF/93.01 - Aprobar el uso obligatorio del Plan Contable Gubernamental 2009 y sus modificatorias; Resolución Consejo Normativo de Contabilidad No 043-2010-EF/94 - Aprobar la versión modificada del Plan Contable General Empresarial. El saneamiento contable en el sector público, alcanza a todas las entidades públicas sin excepción, en el marco de sus actividades orgánico funcionales, de acuerdo a lo señalado en el artículo $24^{\circ}$ de la Ley $\mathrm{N}^{\circ} 28708$, Ley General del Sistema Nacional de Contabilidad.

La responsabilidad de las acciones de saneamiento contable recae en los Titulares de las entidades públicas, de acuerdo a lo señalado en el artículo $3^{\circ}$ de la Ley № 29608, quienes dispondrán la creación del Comité de Saneamiento Contable, el cual estará constituido de acuerdo a lo siguiente: el Director General de Administración o cargo equivalente, quien lo presidirá; el Director de Contabilidad o cargo equivalente; el Director de Tesorería o cargo equivalente; el Director de Abastecimiento o cargo equivalente; el Director del Órgano de Control Institucional, si lo hubiere, en calidad de veedor. El Comité de Saneamiento Contable establecerá las acciones que permitan reconocer, clasificar y medir las cuentas contables cuyos saldos actuales ameriten la aplicación de las acciones de saneamiento contable.

\section{ANTECEDENTES DEL SANEAMIENTO:}

Interpretando a Paredes, D. (2013), en los informes de auditoría a los estados financieros del ejercicio 2010 y de ejercicios anteriores, realizados a las entidades públicas, se observó que existen dictámenes conteniendo "opinión con salvedad", "opinión adversa" o "abstención de opinión”, basados principalmente en aspectos relacionados con el saneamiento físico, legal y contable de bienes de propiedad, planta y equipo. Existen otros rubros observados en menor proporción, como las Existencias, Cuentas por Cobrar, Cuentas por Pagar y otras cuentas del activo y pasivo, que vienen afectando los estados financieros por varios años consecutivos.

En ese sentido, la Contraloría General de la República recomendó a la Comisión de Presupuesto y Cuenta General de la República del Congreso, evaluar o proponer iniciativas legislativas que permitan establecer responsabilidades en la presentación de información y evaluar la normativa en saneamiento físico, legal y toma de inventarios de bienes de activo fijo, a fin de plantear modificaciones legislativas. Con ello, se contribuiría a que las entidades públicas cuenten con la documentación que sustente los registros contables de tales rubros y, en tal sentido, el Congreso de la República aprobó la Ley $\mathrm{N}^{\mathrm{o}} 29608$, cuyos artículos $3^{\circ}$ y $4^{\circ}$ disponen el saneamiento contable. Cabe señalar que en años anteriores se emitieron dispositivos legales vinculados a este tema, los cuales se detallan a continuación: Ley No 26512 -"Declaran de Necesidad y Utilidad Pública el Saneamiento Legal de los Inmuebles de Propiedad de los Sectores de Educación, Transportes, Comunicaciones, Vivienda y Construcción" emitida el 21 de julio de 1995; Ley No 27171 -"Ley de Saneamiento y Transferencia de Infraestructura Social financiada por el Fondo Nacional de Compensación y Desarrollo Social-FONCODES" emitida el 11 de setiembre de 1999; Ley No 27493 -"Ley de Saneamiento Físico Legal de Bienes Inmuebles de las Entidades del Sector Público" emitida el 11 de junio de 2001; Ley No 29151 -"Ley General del Sistema Nacional de Bienes Estatales" - SBN emitida el 14 de diciembre de 2007; Decreto de Urgencia No 071-2001 -"Declaran de interés nacional el saneamiento técnico, legal y contable de los inmuebles de propiedad de las entidades públicas" emitido el 18 de junio de 2001; Decreto Supremo N ${ }^{\circ}$ 130-2001-EF-'Dictan medidas reglamentarias para que cualquier entidad pública pueda realizar acciones de saneamiento técnico, legal y contable de inmuebles de propiedad estatal" emitido el 02 de julio de 2001; Decreto Supremo No 136-2001-EF-"Amplían alcances de Reglamento del Saneamiento Técnico, Legal y Contable de los inmuebles de propiedad de las entidades públicas a todos los organismos e instituciones a que se refiere la Ley No 27493" emitido el 09 de julio de 2001; Decreto Supremo $N^{\circ}$ 007-2008-VIVIENDA - Reglamento de la Ley No 29151 Ley General del Sistema Nacional de Bienes Estatales emitido el 14 de marzo de 2008; Resolución No 147-2009/SBN - Aprueba la Directiva N ${ }^{\circ}$ 005-2009/SBN "Saneamiento de los Bienes Muebles de Propiedad Estatal". 
Según Flores, L. (2011), respecto a las políticas administrativas del saneamiento contable, se tiene que los titulares de las entidades, de acuerdo a lo dispuesto por la Ley $\mathrm{N}^{\circ} 29608$, deberán disponer que el personal de la entidad brinde al Comité de Saneamiento y a las áreas responsables del proceso de saneamiento contable -en las cuales se genere información o sucedan hechos que afecten directa o indirectamente la contabilidad de la entidad- las facilidades necesarias para la realización de su labor. Respecto al control de las acciones de saneamiento contable, se tiene que la Contraloría General de la República, en el marco de sus atribuciones, establecerá los lineamientos pertinentes para que los órganos conformantes del Sistema Nacional de Control, en el ámbito de su Jurisdicción, revisen y evalúen los resultados de la depuración contable.

\section{DISCUSIÓN}

La aplicación del instrumento ha sido desarrollada en forma simultánea a la totalidad de los trabajadores de la institución pública que representa la muestra, para indagar los datos requeridos en el estudio.

El cuestionario, instrumento de la técnica de la encuesta, estuvo conformado por 20 interrogantes ordenadas y concernientes a los problemas de investigación, cada una de las interrogantes contaba además con 2 a más alternativas, las cuales figuran suposiciones de aceptación o el rechazo de la interrogante planteada.

Tabla 1. El saneamiento contable de los estados financieros y la de la calidad de la información de la Cuenta General de la República.

\begin{tabular}{|c|c|c|}
\hline Respuesta & Encuestados & $\%$ \\
\hline$\overline{\mathbf{S i}}$ & 26 & $87 \%$ \\
\hline No & 4 & $13 \%$ \\
\hline No sabe/No Opina & 0 & $0 \%$ \\
\hline Total & 30 & $100 \%$ \\
\hline
\end{tabular}

Fuente: Encuesta realizada 2014

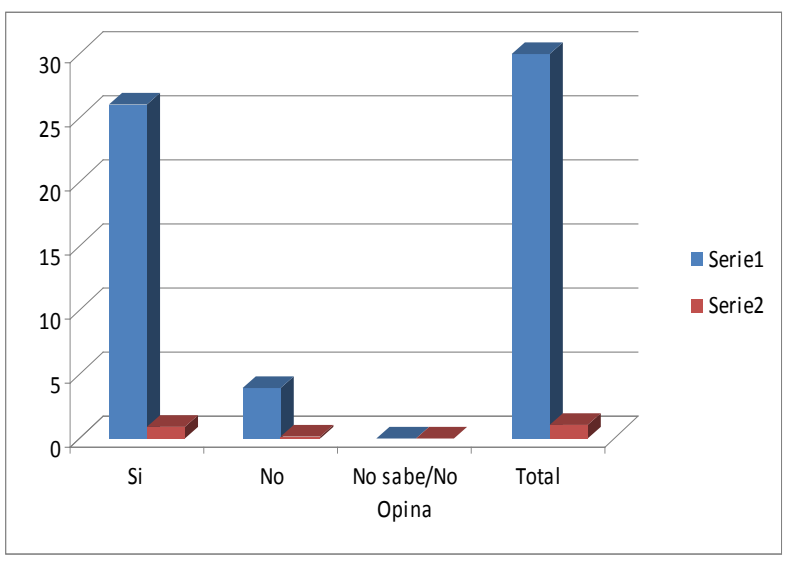

Figura 1. El saneamiento contable de los estados financieros y la de la calidad de la información de la Cuenta General de la República

Fuente: Encuesta realizada 2014

\section{Interpretación:}

Este resultado apoya al saneamiento contable de los estados financieros como procedimiento para lograr la calidad de la información de la Cuenta General de la República. El 87\% de los encuestados acepta que el saneamiento contable de los estados financieros facilita la calidad de la información de la Cuenta General de la República.

Tabla 2. Las Normas Internacionales de Contabilidad para el Sector Público y la presentación adecuada de los resultados de la gestión pública de la Cuenta General de la República.

\begin{tabular}{lcc}
\hline \multicolumn{1}{c}{ Respuesta } & Encuestados & \% \\
\hline Si & 27 & $90 \%$ \\
No & 3 & $10 \%$ \\
No sabe/No Opina & 0 & $0 \%$ \\
Total & $\mathbf{3 0}$ & $\mathbf{1 0 0} \%$ \\
\hline
\end{tabular}

Fuente: Encuesta realizada 2014

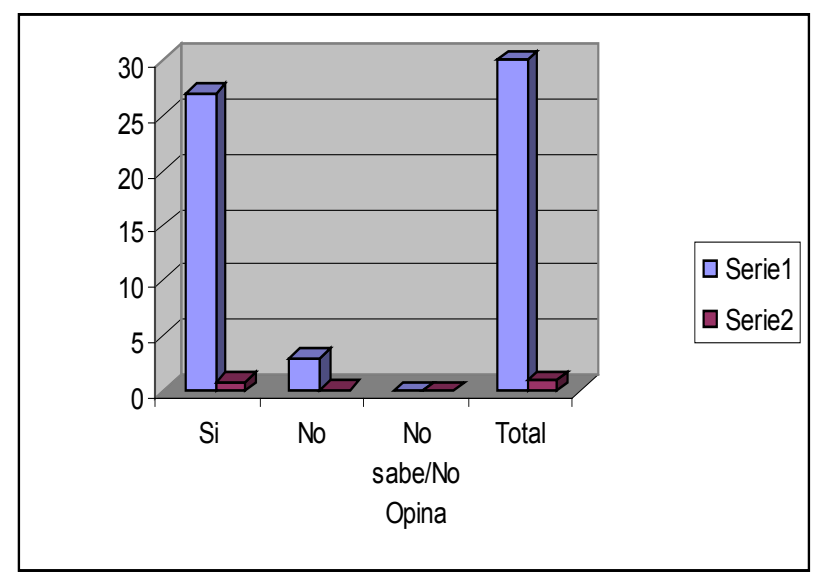

Figura 2. Las Normas Internacionales de Contabilidad para el Sector Público y la presentación adecuada de los resultados de la gestión pública de la Cuenta General de la República.

Fuente: Encuesta realizada 2014

\section{Interpretación:}

Este resultado apoya al saneamiento contable de los estados financieros como procedimiento para lograr la calidad de la información de la Cuenta General de la República. El 90\% de los encuestados acepta que las Normas Internacionales de Contabilidad para el Sector Público facilitan la presentación adecuada de los resultados de la gestión pública de la Cuenta General de la República. 
Tabla 3. Los Clasificadores presupuestarios y la información para el planeamiento de las entidades públicas en la Cuenta General de la República.

\begin{tabular}{lcc}
\hline Respuesta & Encuestados & $\mathbf{\%}$ \\
\hline Si & 29 & $97 \%$ \\
No & 1 & $3 \%$ \\
No sabe/No Opina & 0 & $0 \%$ \\
Total & $\mathbf{3 0}$ & $\mathbf{1 0 0 \%}$ \\
\hline
\end{tabular}

Fuente: Encuesta realizada 2014

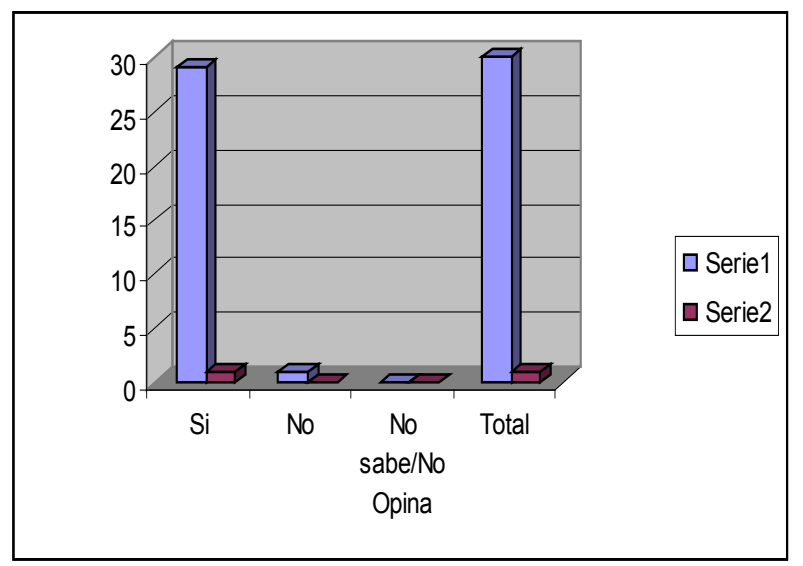

Figura 3. Los Clasificadores presupuestarios y la información para el planeamiento de las entidades públicas en la Cuenta General de la República.

Fuente: Encuesta realizada 2014

\section{Interpretación:}

Este resultado apoya al saneamiento contable de los estados financieros como procedimiento para lograr la calidad de la información de la Cuenta General de la República. El 97\% de los encuestados acepta que los Clasificadores presupuestarios facilitan información para el planeamiento de las entidades públicas en la Cuenta General de la República.

Tabla 4. Las Normas de control gubernamental facilitan el control y la fiscalización de la gestión pública en la Cuenta General de la República.

\begin{tabular}{ccc}
\hline Respuesta & Encuestados & $\%$ \\
\hline $\mathrm{Si}$ & 27 & $90 \%$ \\
No & 3 & $10 \%$ \\
No sabe/No Opina & 0 & $0 \%$ \\
Total & 30 & $100 \%$ \\
\hline
\end{tabular}

Fuente: Encuesta realizada 2014

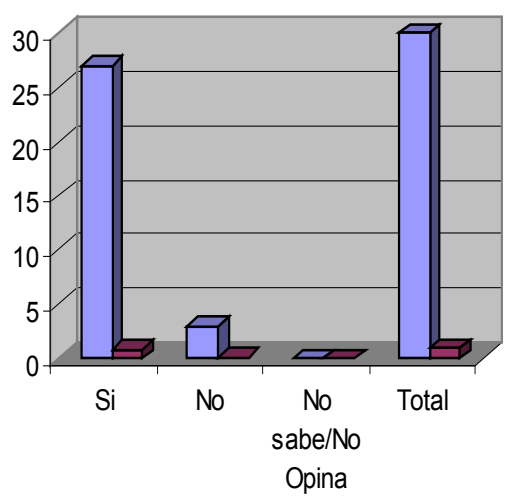

Figura 4. Las Normas de control gubernamental facilitan el control y la fiscalización de la gestión pública en la Cuenta General de la República.

Fuente: Encuesta realizada 2014

\section{Interpretación:}

Este resultado apoya al saneamiento contable de los estados financieros como procedimiento para lograr la calidad de la información de la Cuenta General de la República. El 90\% de lo encuestados acepta que las Normas de control gubernamental facilitan el control y la fiscalización de la gestión pública en la Cuenta General de la República.

TABLA 5. El saneamiento contable tiene su base en los siguientes principios regulatorios de la contabilidad gubernamental: uniformidad; integridad; oportunidad; transparencia y legalidad.

\begin{tabular}{ccc}
\hline Respuesta & Encuestados & \% \\
\hline Si & 29 & $97 \%$ \\
No & 1 & $3 \%$ \\
No sabe/No Opina & 0 & $0 \%$ \\
Total & $\mathbf{3 0}$ & $\mathbf{1 0 0 \%}$ \\
\hline
\end{tabular}

Fuente: Encuesta realizada 2014

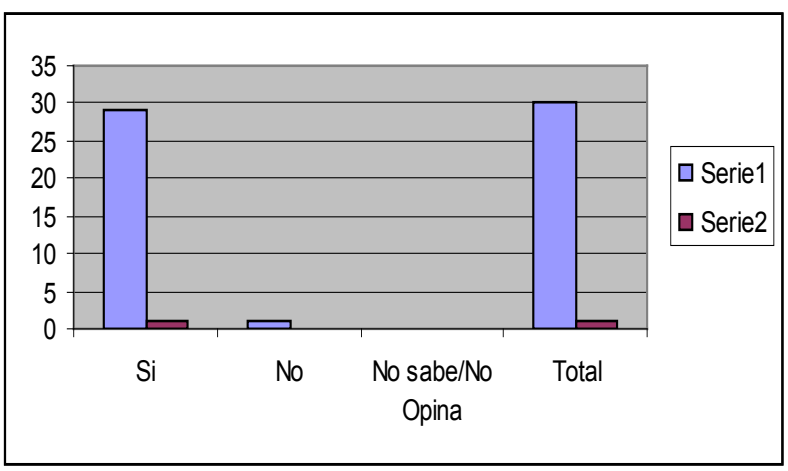

Figura 5. El saneamiento contable tiene su base en los siguientes principios regulatorios de la contabilidad gubernamental: uniformidad; integridad; oportunidad; transparencia y legalidad.

Fuente: Encuesta realizada 2014 


\section{Interpretación:}

Este resultado apoya al saneamiento contable de los estados financieros como procedimiento para lograr la calidad de la información de la Cuenta General de la República; por cuanto el $97 \%$ de los encuestados acepta que el saneamiento contable tiene su base en los siguientes principios regulatorios de la contabilidad gubernamental: uniformidad; integridad; oportunidad; transparencia y legalidad.

Como resultado de la investigación se ha determinado que la contabilidad del sector público se configura como un sistema de información económica, financiera y presupuestaria de cada una de las entidades que lo conforman.

El saneamiento contable de los estados financieros facilita la calidad de la información de la Cuenta General de la República; mediante los ajustes y regularizaciones en el marco de las NIC-SP, Manual de estadísticas públicas, Clasificadores presupuestarios y Normas de control gubernamental. Las Normas Internacionales de Contabilidad para el Sector Público (NIC-SP), y las Normas Internacionales de Información Financiera facilitan la presentación adecuada de los resultados de la gestión pública de la Cuenta General de la República; mediante los procedimientos de saneamiento contable establecidos por el ente rector de la Contabilidad en el Perú. Las Normas de control gubernamental facilitan el control y la fiscalización de la gestión pública en la Cuenta General de la República; mediante la realización de acciones y procedimientos establecidos en la Ley del Sistema Nacional de Control para estos fines.

\section{Literatura citada}

Aldave, J. \& Meniz, A. 2013. Auditoría y control gubernamental. Peru, Lima. Editora Gráfica Bernilla. $136 \mathrm{p}$.

Alvarado, J. 2013. Contabilidad gubernamental. Peru, Lima. Editorial San Marcos. 125p.

Cepeda, G. 2012. Auditoría gubernamental y control interno. Colombia, Bogotá. Editorial Mc Graw Hill. 94p.

Flores, L.2011. Contabilidad Pública para la toma de decisiones. México, DF, Editorial: Trillas. 85p.

Hernández, D. 2007. Desconcentración de la Cuenta General para facilitar el desarrollo, integral y sostenible de las regiones. Tesis Ph.D. Universidad Nacional Federico Villarreal.194 p.

Paredes, D. 2013. Contabilidad y gestión pública. Perú, Lima. Editorial ARPES. 124p. 